\title{
A United Network for Organ Sharing analysis of heart transplantation in adults with congenital heart disease: Outcomes and factors associated with mortality and retransplantation
}

\author{
Tara Karamlou, MD, MSc, ${ }^{\text {a,b }}$ Jennifer Hirsch, MD, MS, ${ }^{a}$ Karl Welke, MD, MS, ${ }^{\mathrm{b}}$ Richard G. Ohye, MD, ${ }^{\mathrm{a}}$ \\ Edward L. Bove, MD, ${ }^{a}$ Eric J. Devaney, MD, ${ }^{a}$ and Robert J. Gajarski, $\mathrm{MD}^{\mathrm{c}}$
}

Objectives: Heart transplantation in patients with adult congenital heart disease is increasing, yet no large studies
have defined how this subgroup differs from other adult recipients. We investigated outcomes and risk factors for
mortality and retransplantation among patients with adult congenital heart disease compared with adult recipients.

Methods: A review was performed of 18- to 45-year-old patients undergoing heart transplantation from 19902008 reported to the United Network for Organ Sharing database. Trends were compared between 2 eras: era 1 (1990-1998) and era 2 (1999-2008). Multivariable semiparametric hazard models identified factors associated with time-related death and retransplantation.

Results: Of 8496 patients identified, 575 had adult congenital heart disease. The prevalence of heart transplantation among adult recipients decreased by $28 \%$ over time $(P<.001)$ and increased among patients with adult congenital heart disease by $41 \%(P<.001)$. Induction therapy use was less in patients with adult congenital heart disease $(66 \%)$ compared with that seen in adult recipients $(71 \%, P=.02)$. Steroid maintenance was less in patients with adult congenital heart disease $(92 \%)$ compared with that seen in adult recipients $(97 \%, P<.001)$. Post-heart transplantation survival among adult recipients improved over time $(P=.02)$ but not among patients with adult congenital heart disease $(P=.81)$. Overall post-heart transplantation mortality $(P=.006)$ and retransplantation $(P=.03)$ were significantly higher for patients with adult congenital heart disease than for adult recipients, mainly because of an early hazard phase. Adult congenital heart disease was a risk factor for both death $(P<.001)$ and retransplantation $(P=.04)$. Any induction therapy and steroid maintenance was associated with improved survival for all recipients $(P=.001)$.

Conclusions: Adult congenital heart disease represent an increasing proportion of heart transplant recipients. Compared with adult recipients, patients with adult congenital heart disease experience higher post-heart transplantation mortality and retransplantation. Immunosuppression differs among patients with adult congenital heart disease and adult recipients. Further studies should investigate whether post-heart transplantation outcomes would be improved by more aggressive induction therapy or judicious steroid tapers. (J Thorac Cardiovasc Surg 2010;140:161-8)

Advances in surgical and medical management have greatly improved the long-term survival of patients with congenital heart disease. ${ }^{1-3}$ However, cardiac dysfunction can occur after palliative or corrective surgery and is the most common cause of late attrition in patients surviving with

\footnotetext{
From the Division of Pediatric Cardiovascular Surgery, ${ }^{\mathrm{a}}$ Section of Cardiac Surgery, Department of Surgery, University of Michigan, Ann Arbor, Mich; the Division of Pediatric Cardiac Surgery, ${ }^{\mathrm{b}}$ Doernbecher Children's Hospital, Oregon Health \& Science University, Portland, Ore; and the Division of Pediatric Cardiology, ${ }^{\mathrm{c}}$ Department of Pediatrics, University of Michigan, Ann Arbor, Mich.

Disclosures: None.

Read at the Thirty-fifth Meeting of the Western Thoracic Surgical Association, Banff, Canada, June 24-27, 2009.

Received for publication June 23, 2009; revisions received March 12, 2010; accepted for publication March 26, 2010; available ahead of print May 3, 2010.

Address for reprints: Tara Karamlou, MD, MSc, Oregon Health Sciences University, Division of Cardiothoracic Surgery, Mail Code L353, 3181 SW Sam Jackson Park Rd, Portland, OR 97239 (E-mail: karamlou@ohsu.edu).

$0022-5223 / \$ 36.00$

Copyright (c) 2010 by The American Association for Thoracic Surgery

doi:10.1016/j.jtcvs.2010.03.036
}

congenital heart disease. ${ }^{4} \mathrm{~A}$ recent study ${ }^{5}$ reported that $20 \%$ of patients with congenital heart disease can have heart failure necessitating heart transplantation (HTx). This patient population presents multiple unique surgical and medical challenges that complicate cardiac transplantation given their complex anatomy, prior surgical and interventional procedures, and frequently increased pulmonary vascular resistance from long-standing congestive heart failure or cyanosis.

Coupled with the added complexity of HTx in patients with adult congenital heart disease (ACHD) compared with other adult recipients (ARs), patients with ACHD are likely to comprise an increasing number of HTx recipients in the near future. ${ }^{1,3,4}$ Many of these are anticipated to be single-ventricle patients, a subgroup whose outcomes might be less favorable than those patients with biventricular physiology. ${ }^{4,6}$ Despite these changes in the ACHD recipient pool, preoperative selection and postoperative management of patients with ACHD and ARs have been similar without evidence supporting this approach. Aside from the annual 


\author{
Abbreviations and Acronyms \\ $\mathrm{ACHD}=$ adult congenital heart disease \\ $\mathrm{AR}=$ adult recipient \\ CHD = congenital heart disease \\ HTx = heart transplantation \\ $\mathrm{RTx}=$ retransplantation \\ UNOS $=$ United Network for Organ Sharing
}

International Society of Heart and Lung Transplantation registry reports, ${ }^{1}$ which include a brief summary of overall outcomes, there are only single-institution reports for posttransplantation outcomes in patients with ACHD. Considering the rapidly increasing population of patients with ACHD, a comparison of posttransplantation management strategies and outcomes to ARs based on cumulative United Network for Organ Sharing (UNOS) experience is timely.

We therefore undertook this study to evaluate current management strategies for ACHD after HTx, to identify determinants associated with mortality and retransplantation (RTx), and to compare these with AR outcomes.

\section{MATERIALS AND METHODS \\ Patients}

Using the UNOS database from 1990-2008, we identified all recipients between the ages of 18 and 45 years undergoing isolated HTx. We chose this age group to capture the vast majority of patients with ACHD and to compose a more homogeneous pool of recipients with more closely approximated demographics and whose outcomes were less likely to be influenced by other medical comorbidities typical of older ARs but rare in patients with ACHD. Patients were grouped into broad diagnostic categories (congenital heart disease, dilated cardiomyopathy, ischemic cardiomyopathy, restrictive cardiomyopathy, and other), as were immunosuppression regimens to simplify analyses (Appendices 1 and 2). Patients without follow-up information $(\mathrm{n}=583)$ were excluded.

\section{Statistical Analysis}

Overall descriptive statistics were computed. Recipient groups (ARs and patients with ACHD) were compared by using the $\chi^{2}$ test for categorical variables or the $t$ test for continuous variables. Percentages were calculated by using the number of available data, with missing values indicated. Trends were compared between 2 eras, era 1 (1990-1998) and era 2 (1999-2008), to capture changes in immunosuppression (ie, introduction of mycophenolate mofetil and transition from cyclosporine to tacrolimus). Time-related death and RTx were computed by using the Kaplan-Meier method, and the log-rank statistic was used to determine differences between groups. Multivariable semiparametric hazard models identified factors associated with time-related death and RTx. SAS software, version 9.1 (SAS Institute, Inc, Cary, NC), was used for all analyses. Institutional review board approval was obtained, but given the deidentified nature of the UNOS database, patient consent was waived for this study.

\section{RESULTS}

\section{Case Mix}

Demographics and clinical characteristics of the study patients are shown in Table 1. Of 8496 cases identified, 6.8\% $(\mathrm{n}=575)$ were patients with ACHD, and 93.2\% $(\mathrm{n}=7921)$ were ARs. The AR group was comprised of the following diagnostic subgroups: dilated cardiomyopathy, $46.4 \%$ $(\mathrm{n}=3939)$; inoperable coronary artery disease (nondilated ischemic cardiomyopathy), $19.1 \%(\mathrm{n}=1624)$; hypertrophic cardiomyopathy, $2.5 \%(\mathrm{n}=215)$; valvular heart disease, $2.7 \%(\mathrm{n}=231)$; and restrictive cardiomyopathy, $1.9 \%$ $(\mathrm{n}=163)$. Patients with ACHD were younger, had longer ischemic and waitlist times, and were more likely to have preoperative extracorporeal membrane oxygenation support $(P<.05$ for all). In contrast, status 1 prevalence was higher among ARs than patients with ACHD $(P<.001)$. Between eras 1 and 2, the frequency of HTx decreased by $28 \%$ in ARs ( 4033 to $2888, P<.001$ ) but increased by $41 \%$ among patients with ACHD (239 to 336, $P<.001)$.

\section{Immunosuppression Regimen}

Induction therapy. Immunosuppression regimens among groups are shown in Table 1. Overall use of induction therapy with standard agents, including antithymocyte globulin, Muromonab-CD3, and interleukin 2 receptor antagonists, was less prevalent in patients with ACHD (66.5\%) compared with that seen in ARs $(71.2 \%, P=.02)$. In particular, patients with ACHD were less likely than ARs to receive steroid induction $(72.8 \%$ vs $77.6 \%, P=.01)$ without a corresponding increase in alternative agents (Figure 1). In general, induction therapy was less prevalent among both groups over time; however, this might represent an underreporting of newer agent use, including interleukin 2 antagonists, within the UNOS database.

Maintenance therapy. Maintenance therapy was similar among patients with ACHD and ARs, except that steroids were less frequently used in patients with ACHD (92.1\%) than in ARs $(96.6 \%, P<.001)$. Maintenance regimens also changed over time in both groups. Decreased use of steroids in patients with ACHD relative to ARs persisted in both eras (Figure 1). Tacrolimus replaced cyclosporine as the predominant calcineurin inhibitor, and azathioprine was used more frequently for maintenance in patients with ACHD than in ARs $(86.8 \%$ vs $72.2 \%, P=.02)$.

\section{Mortality and RTx}

There were 3208 total deaths during the study period, yielding an overall mortality of $37.8 \%$. Cause of death was graft failure in 665 patients, cardiovascular failure in 733 patients, infection in 271 patients, malignancy in 156 patients, pulmonary failure in 81 patients, hemorrhage in 65 patients, technical in 8 patients, other in 492 patients, and unknown in 737 patients. Cause of death was similar among both groups, except patients with ACHD had a greater prevalence of death from early hemorrhage $(\mathrm{n}=15[2.6 \%])$ compared with ARs $(\mathrm{n}=76[1 \%], P<.001)$, and ARs had a greater prevalence of death from malignancy $(\mathrm{n}=152$ [2.1\%]) compared with patients with ACHD ( $\mathrm{n}=4$ 
TABLE 1. Demographic characteristics among study patients

\begin{tabular}{|c|c|c|c|}
\hline Variable & Patients with ACHD $(n=575)$ & $\operatorname{ARs}(n=7921)$ & $P$ value \\
\hline Age $(y)$, mean $\pm S D$ & $28.3 \pm 8.1$ & $35.4 \pm 7.9$ & $<.001$ \\
\hline Female sex, no. $(\%)$ & $212(37)$ & $2476(31)$ & .005 \\
\hline Status 1 , no. $(\%)$ & $355(62)$ & $5809(73)$ & $<.001$ \\
\hline Waitlist time (d), median (range) & $114(0-2245)$ & $74(0-4318)$ & $<.001$ \\
\hline Ischemic time (h), median (range) & $3.8(1-8.9)$ & $2.9(0.5-12)$ & $<.001$ \\
\hline ECMO before transplantation, no. $(\%)$ & $6(1)$ & $34(0.4)$ & .04 \\
\hline \multicolumn{4}{|l|}{ Immunosuppression regimens, no. $(\%)$} \\
\hline Steroid induction & $385(72.8)$ & $5268(77.6)$ & .01 \\
\hline OKT3 induction & $56(74.7)$ & $600(71.2)$ & .52 \\
\hline Induction with any agent & $358(66.5)$ & $5191(71.2)$ & .02 \\
\hline Cyclosporine maintenance & $119(99.2)$ & $2074(98.9)$ & .36 \\
\hline Azathioprine maintenance & $216(90.8)$ & $3294(91.6)$ & .65 \\
\hline Steroid maintenance & $487(92.1)$ & $7010(96.6)$ & $<.001$ \\
\hline
\end{tabular}

$A C H D$, Adult congenital heart disease; ARs, adult recipients; $S D$, standard deviation; ECMO, extracorporeal membrane oxygenation; OKT3, Muromonab-CD3.

$[0.7 \%], P=.02)$. Freedom from death at 1,10 , and 15 years for the entire study population was $86 \%, 54 \%$, and $38 \%$, respectively (Figure 2), with similar crude mortality between groups (227/575 [39\%] vs 2981/7921 [38\%], $P=.9$ ). Importantly, post-HTx survival among ARs improved over time (era 1: 1 - and 5-year survival of $85 \%$ and $67 \%$, respectively; era $2: 1-$ and 5-year survival of $87 \%$ and $71 \%$, respectively; $P=.02$ ), whereas no era effect was seen among patients with ACHD (era 1: 1- and 5-year survival of $76 \%$ and $63 \%$, respectively; era $2: 1$ - and 5 -year survival of $75 \%$ and $63 \%$, respectively; $P=.81$; Figure 3). KaplanMeier freedom from death was significantly higher for ARs (1- and 10-year survival of $87 \%$ and $55 \%$, respectively) than for patients with ACHD (1- and 10-year survival of 76\% and $52 \%$, respectively, $P=.003$ ), mainly because of an early hazard phase (Figure 4).

Multivariable factors associated with increased mortality included ACHD $(P<.001)$, younger age $(P=.002)$, status $1(P=.03)$, longer ischemic times $(P<.001)$, and female sex $(P=.04)$. Any induction therapy $(P=.008)$ and steroid maintenance therapy $(P<.001)$ were associated with improved survival for all recipients, and there was an impor- tant interaction term whereby patients with ACHD gained an additional survival benefit with use of maintenance steroids $(P<.001$, Table 2$)$.

For the overall cohort, RTx was necessary in $273(3.5 \%)$ patients. There was a higher RTx rate in patients with ACHD $(\mathrm{n}=27 / 575[4.7 \%])$ than in ARs $(\mathrm{n}=266 / 7921[3.4 \%]$, $P=.09$ ). Kaplan-Meier freedom from RTx was greater for ARs (1- and 10-year freedom of $99 \%$ and $91 \%$, respectively) than for patients with ACHD (1- and 10-year freedom of $96 \%$ and $86 \%$, respectively; $P=.04$; Figure 5). Multivariable factors associated with an increased risk of RTx included ACHD $(P=.03)$.

\section{DISCUSSION}

We have shown, using a large administrative database, that mortality and RTx rates are higher after HTx for ACHD than those for patients with other underlying diagnoses. Although ARs have seen an improvement in these outcomes over time, no era effect could be demonstrated for patients with ACHD, mainly because of the persistent early attrition among patients with ACHD in the first year after transplantation. Importantly, although patients with ACHD are still a small

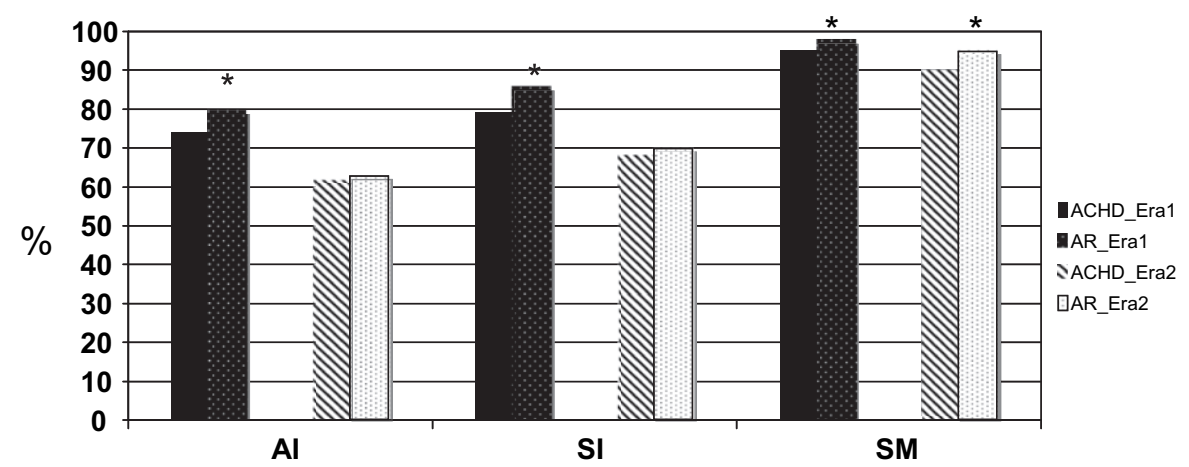

FIGURE 1. Immunosuppression trends over time among patients with adult congenital heart disease $(A C H D)$ and adult recipients $(A R s)$. In era 1 patients with ACHD significantly received less aggressive induction than ARs. In era 2, although the induction gap decreased among groups, the prevalence of steroid maintenance was still significantly lower in patients with ACHD compared with that seen in ARs. AI, Any induction with standard agents excluding steroids; $S I$, steroid induction; $S M$, steroid maintenance. $* P<.05$. 


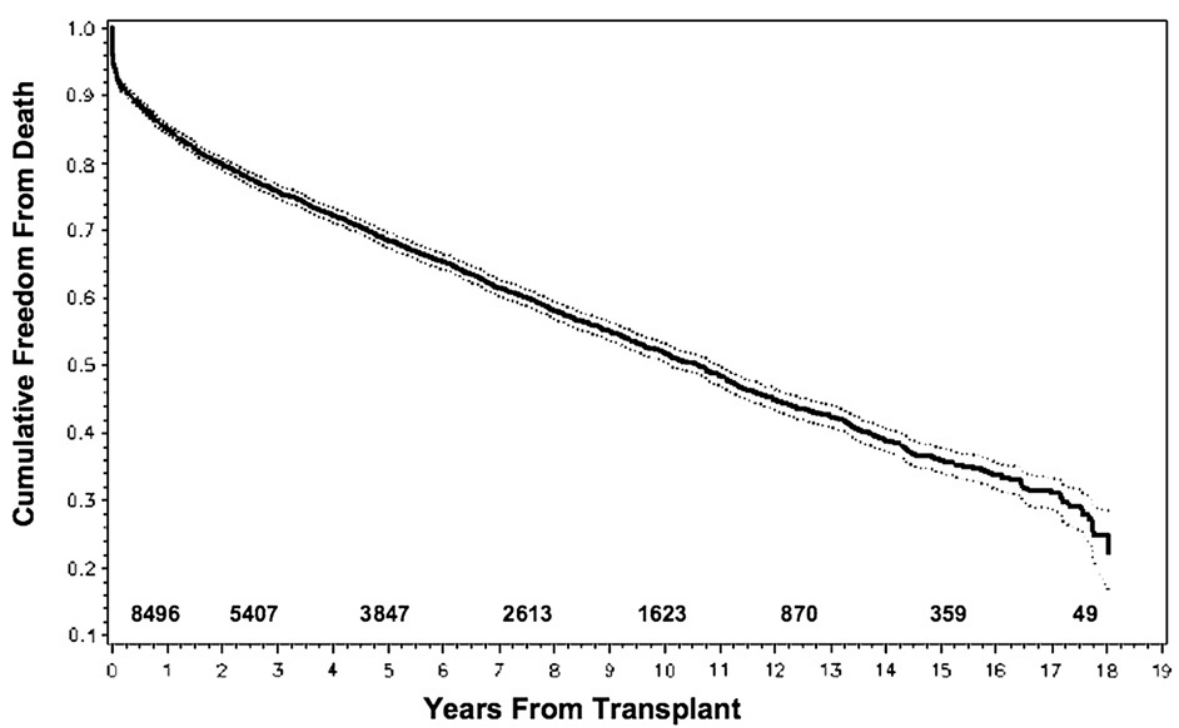

FIGURE 2. Overall risk-unadjusted freedom from death after transplantation. Numbers at bottom inset show the number of patients remaining at risk. Dashed lines enclose $95 \%$ confidence intervals.

overall proportion of the recipient pool (approximately $2 \%$ ), the incidence of HTx is increasing among patients with ACHD in the recent era compared with ARs, in whom HTx incidence has decreased. Certainly, inherent differences among these groups, such as age, which we found to be a risk factor for mortality, explain some of the variation in post-HTx death and RTx. However, our study is unique in that we have elucidated differences in immunosuppression regimens among patients with ACHD and ARs that could represent modifiable therapeutic targets. Specifically, more aggressive induction therapy and use of steroid maintenance therapy in patients with ACHD could lead to improved outcomes.

Our data agree with the 24th Official Adult Heart Transplant Report by Taylor and colleagues, ${ }^{1}$ which showed that an underlying diagnosis of ACHD was a powerful risk factor for 1-year mortality, with a 2- to 3-fold increase in relative risk. Pigula and associates ${ }^{6}$ also found in a subanalysis of 8 patients (of 33 undergoing combined cardiopulmonary transplantation) that patients with ACHD had significantly worse survival after HTx than ARs. The influence of underlying diagnosis of ACHD has been negligible in other studies. ${ }^{2,3}$ Lamour and coworkees ${ }^{2}$ studied 24 patients older than 18 years with an underlying diagnosis of congenital heart disease (CHD) who underwent HTx at their institution from 1995-1998. These authors found similar 1-year survival $(75 \%)$ among patients with ACHD to that reported in our present study. However, post-HTx 1-year survival among a control group in their study, which was composed of 33 ARs, was only $79 \%$, and they concluded that outcomes for ARs and patients with ACHD are equivalent. $^{2}$ The Mayo Clinic reported excellent outcomes, with 16 patients having CHD, 11 of whom were older than 18 years. ${ }^{3}$
Actuarial survival was $86 \%$ at 5 years, although, similar to our findings, these authors also noted an early hazard phase for the patients with ACHD after HTx. Importantly, in the Mayo Clinic series patients with CHD received aggressive induction with steroids, OKT3, and azathioprine, and they were maintained on prednisone $(0.2 \mathrm{mg} / \mathrm{kg})$ daily. Our data would support that outcomes in patients with ACHD could be improved by administering aggressive induction and continued judicious use of steroids after HTx.

The lack of improved outcomes over time among patients with ACHD, as opposed to ARs, is an important finding in our analysis. The negligible era effect is mainly because of the unmitigated early attrition among patients with ACHD, which can be attributed to challenges within preoperative, postoperative, and intraoperative care. ${ }^{3,4,7} \mathrm{We}$ recognize that our findings might seem contradictory in that induction use and steroid maintenance decreased over time in both groups, yet outcomes improved within ARs. However, it is possible that we have failed to capture increased use of newer induction agents, including interleukin 2 receptor antagonists, which are underreported in our dataset. It is also likely that the early hazard among patients with ACHD is due to more complex perioperative factors that have yet to be elucidated. Selection of patients with ACHD with increased pulmonary vascular resistance for isolated cardiac rather than combined cardiopulmonary transplantation can be challenging. Many single-ventricle recipients with failing Fontan circulation have systemic complications, such as protein-losing enteropathy or systemic arteriovenous or venovenous collaterals, which can complicate intraoperative management and postoperative care. Most recipients with ACHD have also had prior cardiac surgery, and many have complex anatomy, both of which 


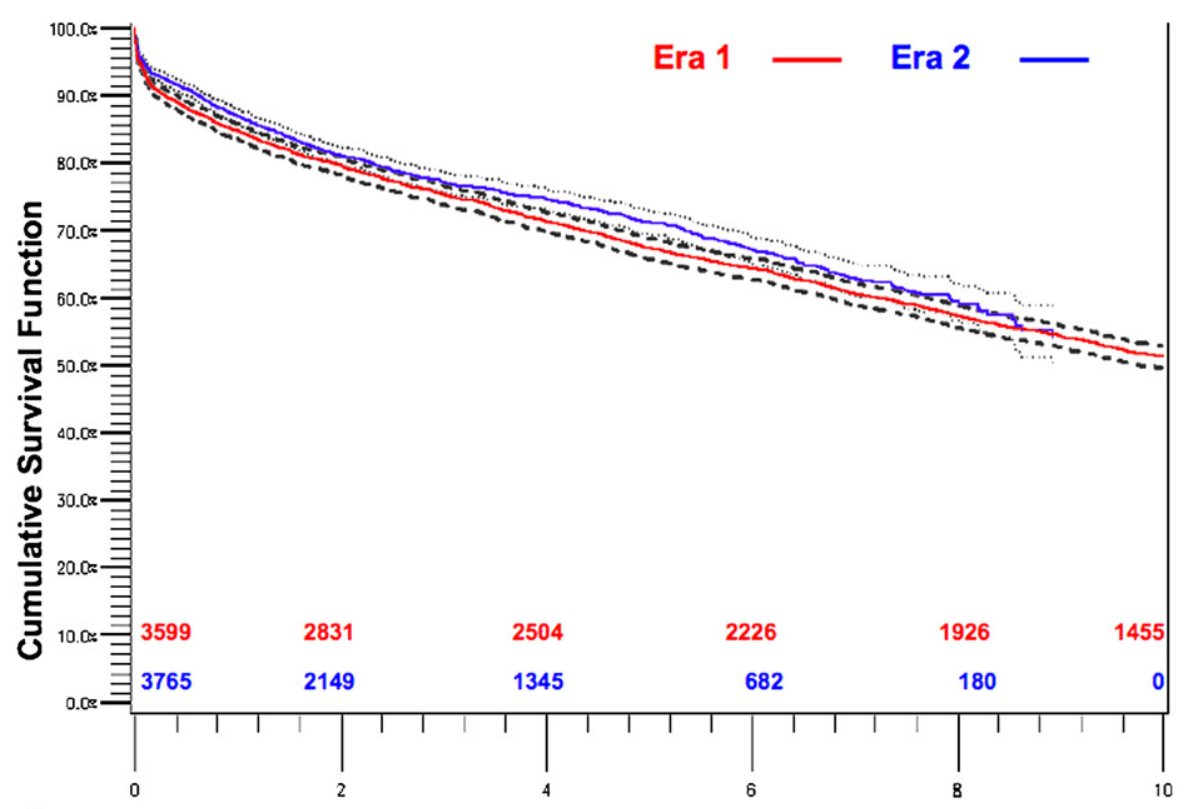

A

Years From Transplant

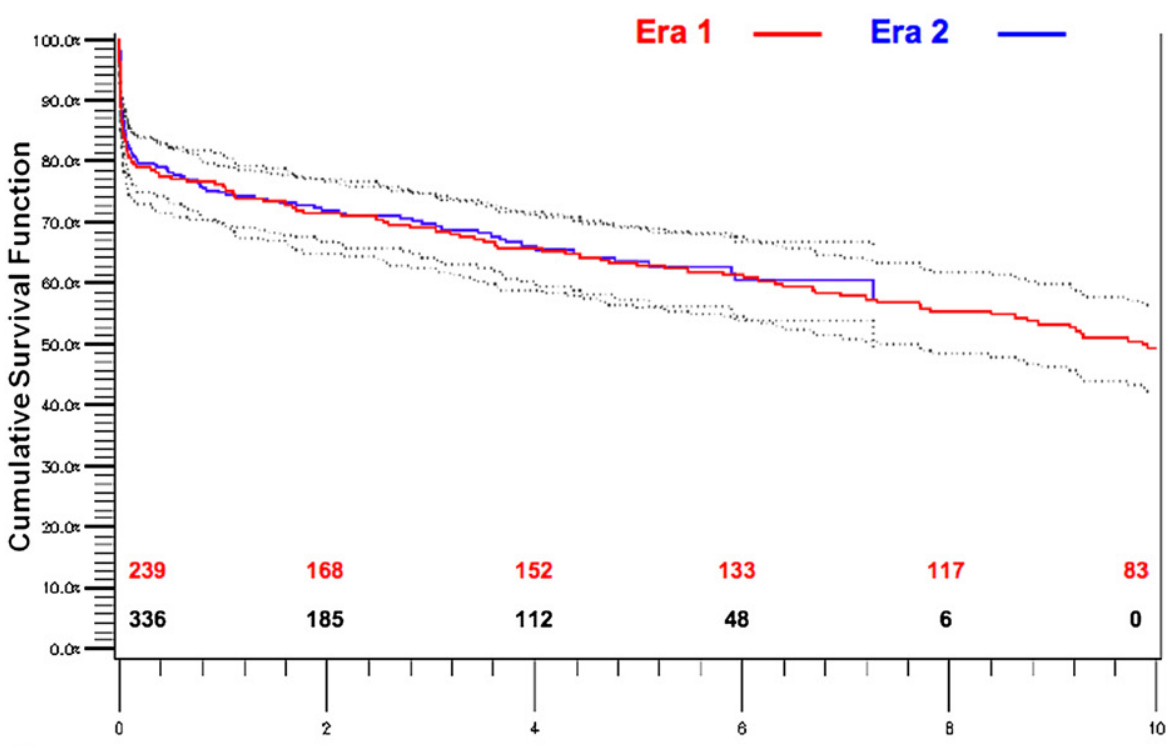

B

Years From Transplant

FIGURE 3. A, Risk-unadjusted freedom from death for adult recipients stratified by eras demonstrates an improvement in mortality over time, especially within midterm survival. Note the areas of the curves between years 4 and 7 in which the confidence limits do not overlap. Era 1 (1990-1998) is depicted in red, and era 2 (1999-2008) is depicted in blue. Solid circles represent censored patients. Numbers at inset show the number of patients remaining at risk. Dashed lines enclose $95 \%$ confidence intervals. B, Risk-unadjusted freedom from death for patients with adult congenital heart disease was equivalent in both eras. Era 1 (1990-1998) is depicted in red, and era 2 (1999-2008) is depicted in blue. Solid circles represent censored patients. Numbers at inset show the number of patients remaining at risk. Dashed lines enclose $95 \%$ confidence intervals.

necessitate longer cardiopulmonary bypass and ischemic times and contribute to postoperative coagulopathy and increased infection risk. ${ }^{4,7}$

Certain factors that likely contribute to the difference in posttransplantation outcomes among patients with ACHD and ARs, including the precise congenital diagnosis, the prevalence and distribution of recipient comorbidities, and the number of prior cardiac procedures, could not be captured with the current dataset. Further elaboration of the influence of these variables using either the International Society for Heart and Lung Transplantation database or a more complete single-institution database is warranted.

However, our data suggest that a potentially modifiable reason underlying the increased risk of death among 


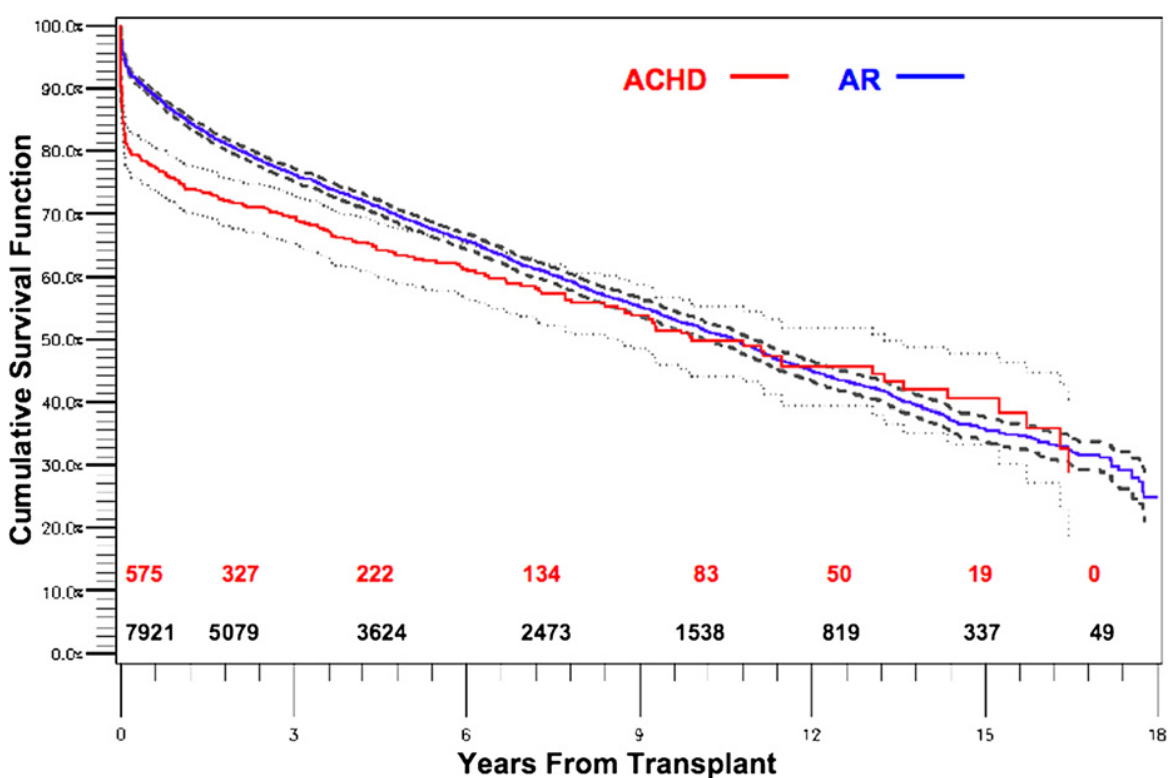

FIGURE 4. Risk-unadjusted freedom from death stratified by underlying diagnosis, either patients with adult congenital heart disease ( $A C H D$ ) or adult recipients (ARs). The blue line depicts ARs, and the red line depicts patients with ACHD. Survival is significantly better for ARs than for patients with ACHD, mainly because of an early hazard phase representing increased postoperative mortality for patients with ACHD that persists to 2.5 years after transplantation. Numbers at inset show the patients remaining at risk. Dashed lines enclose $95 \%$ confidence intervals.

patients with ACHD is a difference in postoperative immunosuppression. Patients with ACHD were less likely to receive induction therapy and were less likely to be treated with maintenance steroids. The risk of allograft rejection is highest in the first several months after transplantation, and cytolytic induction therapy, which rapidly depletes recipient lymphocytes, might mitigate the risk of acute rejection. Patients with ACHD might be at increased risk for rejection because of increased levels of lymphocytotoxic IgG class antibodies from multiple prior blood transfusions or use of homograft implants. ${ }^{4,8,9}$ Unfortunately, data regarding early rejection episodes were available in too few patients within the UNOS dataset to allow a meaningful analysis, although this will be an important focus for future study.

TABLE 2. Multivariable factors associated with posttransplantation mortality

\begin{tabular}{lccc}
\hline \multicolumn{1}{c}{ Variable } & $\begin{array}{c}\text { Parameter } \\
\text { estimate }( \pm \mathbf{S E})\end{array}$ & $\begin{array}{c}\text { Hazard } \\
\text { ratio }\end{array}$ & $\boldsymbol{P \text { value }}$ \\
\hline ACHD & $0.67 \pm 0.15$ & 1.96 & $<.001$ \\
Younger age & $0.01 \pm 0.002$ & 1.01 & .003 \\
Female sex & $0.08 \pm 0.04$ & 1.10 & .03 \\
Longer ischemic time & $0.07 \pm 0.02$ & 1.07 & $<.001$ \\
No steroid maintenance & $0.78 \pm 0.09$ & 2.18 & $<.001$ \\
No induction agent & $0.19 \pm 0.07$ & 1.22 & $<.001$ \\
Status 1 & $0.09 \pm 0.04$ & 1.09 & .03 \\
Interaction term between ACHD & - & 0.51 & $<.001$ \\
$\quad$ and steroid maintenance & & & \\
\hline$S E$ Stan
\end{tabular}

$S E$, Standard error; $A C H D$, adult congenital heart disease.
We also found that younger age and longer ischemic time were risk factors for death. The 2007 International Society of Heart and Lung Transplantation report similarly detailed that extremes of age ( $<20$ and $>60$ years), as well as longer duration of ischemic time, were associated with an increased risk of death. ${ }^{1}$ We anticipated that advanced age would be an influential variable and therefore chose a study population between 18 and 45 years to reduce this bias. It is unclear what underlies the difference in postoperative immunosuppression therapy among patients with ACHD and ARs. One possibility is that health care providers are reticent to administer steroid maintenance in patients with ACHD because of concerns for late osteoporosis, avascular femoral head necrosis, and accelerated coronary vasculopathy. A less aggressive approach to induction therapy might similarly belie an unfamiliarity regarding the possible effect of newer medications on younger patients. We were not able to gather data regarding the specialty (eg, pediatric cardiologists vs adult cardiologists) of post-HTx providers, but prior reports, albeit not specific to HTx recipients, have demonstrated that patients with ACHD fare better when cared for within a children's hospital environment by pediatric specialists who are more familiar with the complex anatomy and physiology of these challenging patients..$^{10,11}$

\section{Limitations}

Our study has several limitations, including all of those that apply to any retrospective analysis of prospectively collected data. Missing data regarding important outcomes, including early rejection and immunosuppression regimens, 


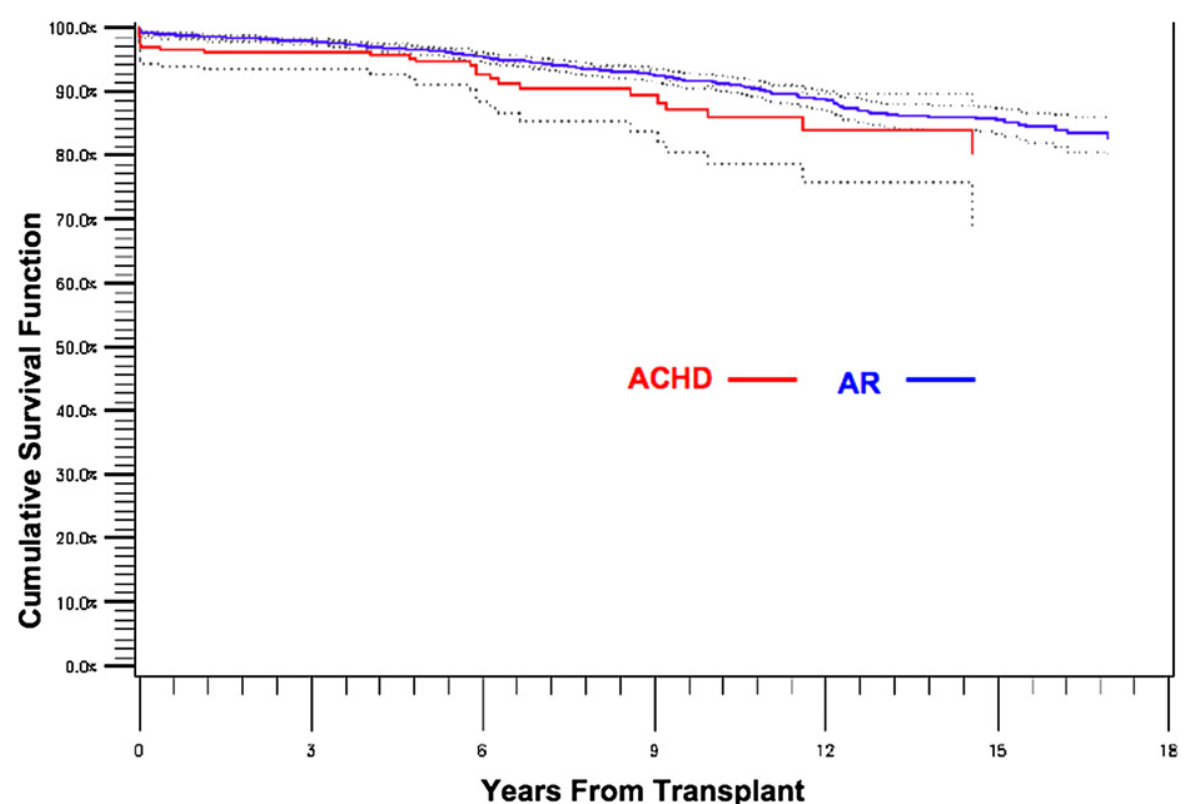

FIGURE 5. Risk-unadjusted freedom from retransplantation stratified by underlying diagnosis, either patients with adult congenital heart disease ( $A C H D$ ) or adult recipients $(A R)$. The blue line depicts ARs, and the red line depicts patients with ACHD. Although the difference is small, note the nearly overlapping confidence intervals, the retransplantation rate is significantly higher for patients with ACHD than for ARs. Dashed lines enclose $95 \%$ confidence intervals.

especially newer agents introduced in the recent era, might have biased our results. Data regarding panel-reactive antibody levels could be crucial to identifying additional inherent differences among patients with ACHD and ARs but were incompletely captured within the UNOS dataset. More detailed information regarding the precise congenital diagnosis, irrespective of whether death was related to graft rejection, the incidence of chronic rejection, and the prevalence and distribution of comorbidities, would also have contributed substantially to the study but is currently unavailable within our dataset. Finally, an analysis of outcomes based on center volume and provider specialty, data unavailable in the present dataset, would have provided more insight underlying the differences in management among patients with ACHD and ARs.

\section{CONCLUSIONS}

Patients with ACHD represent an increasing proportion of HTx recipients. Higher post-HTx mortality and RTx rates among patients with ACHD compared with ARs are persistent over time. Management of immunosuppression differs among patients with ACHD and ARs. Further studies should investigate whether post-HTx outcomes would be improved by more aggressive induction therapy or judicious steroid tapers.

\section{References}

1. Taylor DO, Edwards LB, Boucek MM, Trulock EP, Aurora P, Christie J, et al Registry of the International Society for Heart and Lung Transplantation: Twenty-fourth Official Adult Heart Transplant Report-2007. J Heart Lung Transplant. 2007;26:769-81.

2. Lamour JM, Addonizio LJ, Galantowicz ME, Quaegebeur JM, Mancini DM, Kichuk MR, et al. Outcome after orthotopic cardiac transplantation in adults with congenital heart disease. Circulation. 1999;100(suppl II):II200-5.

3. Speziali G, Driscoll DJ, Danielson GK, Julsrud PR, Porter CJ, Dearani JA, et al Cardiac transplantation for end-stage congenital heart defects: the Mayo Clinic experience. Mayo Clin Proc. 1998;73:923-8.

4. Hosseinspur AR, Cullen S, Tsang VT. Transplantation in adults with congenital heart disease. Eur J Cardiothorac Surg. 2006;30:508-14.

5. Penkoske P, Freedom R, Rowe R, Trusler G. The future of heart and heart-lung transplantation in children. Heart Transplant. 1984;3:233-8.

6. Pigula FA, Gandhi SK, Ristich J, Stukus D, McCurry K, Webber SA, et al. Cardiopulmonary transplantation for congenital heart disease in the adult. J Heart Lung Transplant. 2001;20:297-303.

7. Goerler H, Simon A, Gohrbandt B, Hagl C, Oppelt P, Weidemann J, et al. Heartlung and lung transplantation in grown-up congenital heart disease: long-term single centre experience. Eur J Cardiothorac Surg. 2007;32:926-31.

8. Shaffer KM, Denfield SW, Schowengerdt KO, Towbin JA, Radovancević B, Frazier $\mathrm{OH}$, et al. Cardiac transplantation for pediatric patients with inoperable congenital heart disease. Texas Heart Inst J. 1998;25:57-63.

9. Bailey LL. Transplantation is the best treatment for hypoplastic left heart syndrome. Cardiol Young. 2004;14(suppl I):109-11.

10. Karamlou T, Diggs BS, Person T, Ungerleider RM, Welke KF. National practice patterns for management of adult congenital heart disease: operation by congenital surgeons decreases in-hospital death. Circulation. 2008;118:2345-52.

11. Karamlou T, Diggs BS, Ungerleider RM, Welke KF. Adults or big kids: what is the ideal clinical environment for management of grown-up patients with congenital heart disease? Ann Thorac Surg. 2010. In press. 
APPENDIX 1. UNOS database codes used to define diagnostic groups

ACHD: 1203, 11205, 1206, 1207, 1500, 1501, 1502, 1548, 1549, 1600

Dilated cardiomyopathy: $1000, * 1001,1002,1003,1004,1005,1006$, $1007 \dagger$

Nondilated ischemic cardiomyopathy: 1200

Hypertrophic cardiomyopathy: 1201

Valvular heart disease: 1202

Restrictive cardiomyopathy: 1050, 1051, 1052, 1053, 1054, 1099

Failed prior heart transplantation: 1100, 1101, 1102, 1103, 1104, 1105, 1106, 1199

$\overline{A C H D \text {, Adult congenital heart disease. }{ }^{*} \text { Code for idiopathic dilated cardiomyopathy. }}$ $\dagger$ Code for ischemic dilated cardiomyopathy.

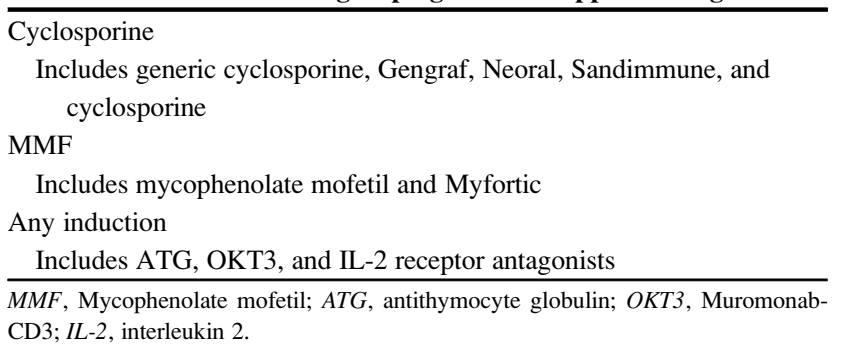

